\title{
Ising Superconductivity and Magnetism in $\mathrm{NbSe}_{2}$
}

\author{
Darshana Wickramaratne (1) \\ Centerfor Computational Materials Science, U.S. Naval Research Laboratory, Washington, DC 20375, USA \\ Sergii Khmelevskyi \\ Research Center for Materials Science and Engineering, Vienna University of Technology, \\ Karlsplatz 13, 1040 Vienna, Austria \\ Daniel F. Agterberg \\ Department of Physics, University of Wisconsin, Milwaukee, Wisconsin 53201, USA \\ I. I. Mazin $\odot$ \\ Department of Physics and Astronomy, George Mason University, Fairfax, Virginia 22030, USA \\ and Quantum Science and Engineering Center, George Mason University, Fairfax, Virginia 22030, USA
}

(Received 11 May 2020; revised 28 June 2020; accepted 28 July 2020; published 5 October 2020)

\begin{abstract}
Recent studies on superconductivity in $\mathrm{NbSe}_{2}$ have demonstrated a large anisotropy in the superconducting critical field when the material is reduced to a single monolayer. Motivated by this recent discovery, we use density-functional theory (DFT) calculations to quantitatively address the superconducting properties of bulk and monolayer $\mathrm{NbSe}_{2}$. We demonstrate that $\mathrm{NbSe}_{2}$ is close to a ferromagnetic instability, and analyze our results in the context of experimental measurements of the spin susceptibility in $\mathrm{NbSe}_{2}$. We show how this magnetic instability, which is pronounced in a single monolayer, can enable sizable singlet-triplet mixing of the superconducting order parameter, contrary to contemporary considerations of the pairing symmetry in monolayer $\mathrm{NbSe}_{2}$, and discuss approaches as to how this degree of mixing can be addressed quantitatively within our DFT framework. Our calculations also enable a quantitative description of the large anisotropy of the superconducting critical field, using DFT calculations of monolayer $\mathrm{NbSe}_{2}$ in the normal state.
\end{abstract}

DOI: 10.1103/PhysRevX.10.041003

\section{INTRODUCTION}

The transition metal dichalcogenides (TMD) exhibit an astonishing variety of phenomena and phase transitions, which includes charge-density waves (CDWs) [1-5], superconductivity [6], and magnetism [7]. Bulk $2 \mathrm{H}-\mathrm{NbSe}_{2}$, which is one of the canonical transition metal dichalcogenides, exhibits a rich phase diagram, which includes a superconducting and a CDW phase [1]. Superconductivity in bulk $\mathrm{NbSe}_{2}$ has been studied extensively both experimentally $[6,8-11]$ and theoretically $[12,13]$, and the superconducting transition temperature $T_{c}$ has been experimentally identified as $\sim 7 \mathrm{~K}$ [9]. While coupling between the superconducting and CDW order parameters is certainly possible, it was found to be weak in $\mathrm{NbSe}_{2}$ : the

Published by the American Physical Society under the terms of the Creative Commons Attribution 4.0 International license. Further distribution of this work must maintain attribution to the author(s) and the published article's title, journal citation, and DOI.
Subject Areas: Condensed Matter Physics,

Materials Science superconducting phase remains robust while the CDW phase collapses as a function of increasing pressure [1] and as a function of increasing disorder introduced by electron irradiation [14]. Since $\mathrm{NbSe}_{2}$ is also a layered van der Waals material, this has inspired several studies of superconductivity in monolayer $\mathrm{NbSe}_{2}[15-18]$ and several intriguing proposals that seek to exploit proximity induced effects at interfaces between monolayer $\mathrm{NbSe}_{2}$ and magnetic materials [19-22]. Furthermore, while $\mathrm{NbSe}_{2}$ is thus far the canonical example of an Ising superconductor, several theoretical studies on other monolayer TMDs such as $\mathrm{TaS}_{2}, \mathrm{TaSe}_{2}$ [16] and preliminary experimental investigations on monolayer $\mathrm{MoS}_{2}$ and $\mathrm{MoTe}_{2}[23,24]$ have also shown indications of hosting Ising superconductivity.

Monolayer $\mathrm{NbSe}_{2}$, unlike the bulk structure, lacks inversion symmetry, which leads to a large spin-orbit (SO) splitting of the states at the $K$, and its inversion partner $K^{\prime}$, points [25] (there is an additional Fermi surface with states around $\Gamma$, which we also discuss later in this study). The magnitude of the SO splitting is larger than the superconducting order parameter. The zero-magnetic field $T_{c}$ of 
monolayer $\mathrm{NbSe}_{2}$ is $\sim 3 \mathrm{~K}[15,16]$, which is lower than the bulk $T_{c}$. The combination of SO coupling and broken inversion symmetry locks the pseudospins near $K$ and $K^{\prime}$ to be parallel to the $c$ axis of the monolayer. Due to timereversal symmetry, pseudospins at the $K$ and $K^{\prime}$ points are antiparallel, and their energies are degenerate. Hence, the Cooper pairs that form completely break their rotational invariance in spin space. This leads to a novel phenomenon, aptly named Ising superconductivity. One key consequence of this unique pairing is that the superconducting phase survives in the presence of in-plane magnetic fields that considerably exceed the Pauli limit $[15,16]$.

Thus far, theoretical analyses of the superconducting pairing mechanism in monolayer $\mathrm{NbSe}_{2}[26,27]$ have relied on model descriptions of superconductivity in materials that lack inversion symmetry [28-30], loosely based on the band structure calculated from first principles [27]. However, a quantitative description of superconductivity in monolayer $\mathrm{NbSe}_{2}$ is lacking. There is also a lack of consistency between first-principles descriptions of superconductivity in bulk $\mathrm{NbSe}_{2}$ and experimental results. Stateof-the-art first-principles calculations that are usually very accurate for superconductors where the pairing is entirely due to electron-phonon coupling overestimate $T_{c}$ in bulk $\mathrm{NbSe}_{2}$ and isostructural $\mathrm{NbS}_{2}[31,32]$ by a factor of $\sim 3$ and the zero-temperature gap by a factor of $\sim 4$. Furthermore, experimental measurements of the spin susceptibility $\chi_{s}$ in bulk $\mathrm{NbSe}_{2}$ [33] find a $\chi_{s} \sim 3 \times 10^{-4} \mathrm{emu} / \mathrm{mole}$, which, as we show later, considerably exceeds the bare bulk Pauli susceptibility $\chi_{0}$.

Two plausible mechanisms that can be invoked to explain this discrepancy between theory and experiment are the potential role of strong electron-electron interactions and strong spin fluctuations. In Ref. [32], the authors suggested the overestimation of $T_{c}$ in their first-principles calculations can be corrected by accounting for a reduction in the effective mass induced by electron-electron interactions, which they described within the $G W$ approximation. However, reducing the effective mass by a factor of $\left(\mathrm{m}^{*} / \mathrm{m}\right)$ reduces the density of states (DOS) by a factor of $\left(\mathrm{m}^{*} / \mathrm{m}\right)$ and increases the magnitude of the electronphonon matrix element by a factor of $\left(\mathrm{m}^{*} / \mathrm{m}\right)$. Indeed, the DOS is proportional to the one-electron Green's function and the electron-phonon matrix element is proportional to the derivative of the inverse Green's function. Since the electron-phonon coupling constant depends linearly on the DOS and quadratically on the matrix element, reducing the effective mass increases the strength of the electronphonon coupling. Hence, strong electron-electron interaction effects alone do not provide a solution to this discrepancy.

The latter mechanism, which is the role of strong spin fluctuations in $\mathrm{NbSe}_{2}$, has thus far remained unaddressed. Fluctuations in the magnetic moment and magnetic order have been shown to be a source of pairing, or pair breaking, of Cooper pairs in a number of other materials $[34,35]$. Furthermore, strong spin fluctuations can also lead to a sizable Stoner renormalization of $\chi_{0}$. To our knowledge, all theoretical studies of bulk and monolayer $\mathrm{NbSe}_{2}$ at their equilibrium lattice parameters have found the material to be nonmagnetic. However, calculations of monolayer $\mathrm{NbSe}_{2}$ subject to tensile strain exceeding $2 \%$ have predicted a ferromagnetic ground state [36].

This seeming lack of consistency between the various theoretical and experimental results on bulk $\mathrm{NbSe}_{2}$ reported in the literature indicates there is still a need to explore the fundamental properties of $\mathrm{NbSe}_{2}$. For example, if spin fluctuations are operative in $\mathrm{NbSe}_{2}$ it is unclear how this may impact arguably the most interesting aspect of Ising superconductivity in monolayer $\mathrm{NbSe}_{2}$, which is the possibility of a singlet-triplet mixed state. Singlet-triplet mixing of the superconducting order parameter has been attracting a lot of recent attention for a variety of reasons, which includes the ability to achieve spin supercurrents $[30,37]$ and the ability to drive superconducting topological transitions $[30,38]$. While Ising superconductivity is well understood at the phenomenological level, it has never been described on a quantitative level using first-principles calculations. This also precludes a quantitative description of Ising superconductivity, which has been observed in monolayers of several other transition metal dichalcogenides beyond $\mathrm{NbSe}_{2}$ [16,23,24].

In this paper we demonstrate that, indeed, bulk and especially monolayer $\mathrm{NbSe}_{2}$ are close to a magnetic instability. We "translate" the existing model theory that has been developed to analyze superconductivity in materials that lack inversion symmetry and bands split by SO interaction [39], such as monolayer $\mathrm{NbSe}_{2}$, into densityfunctional theory (DFT) parlance, which allows us to develop a quantitative theory of the critical field anisotropy in this material. We use collinear and noncollinear fixedspin moment (FSM) calculations to determine the spin susceptibility of bulk and monolayer $\mathrm{NbSe}_{2}$. Finally, we use the insights obtained from our calculations to discuss possible ramifications on the superconducting order parameter in $\mathrm{NbSe}_{2}$, in particular, the factors that control the scale of the triplet admixture to the order parameter. This knowledge is the first step toward a comprehensive quantitative theory of Ising superconductivity in monolayer $\mathrm{NbSe}_{2}$.

\section{ELECTRONIC STRUCTURE}

The bulk unit cell of $2 \mathrm{H}-\mathrm{NbSe}_{2}$ consists of two monolayers of $\mathrm{NbSe}_{2}$ [Fig. 1(a)] where in a single monolayer the $\mathrm{Nb}$ atoms are in a trigonal prismatic coordination with the $\mathrm{Se}$ atoms. The $\mathrm{Nb}$ atoms in one of the monolayers are vertically above the $\mathrm{Se}$ atoms of the second monolayer [cf. Fig. 1(b)], which leads to a center of inversion that is between the two monolayers of the unit cell. The calculated bulk lattice constants, $a=3.449 \AA$ and $c=12.550 \AA$, are 
(a)

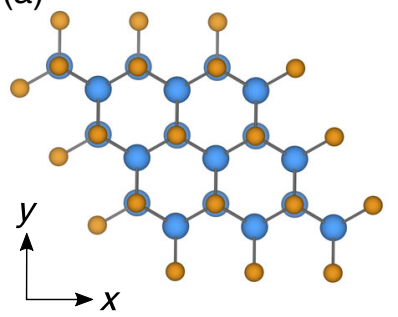

(b)

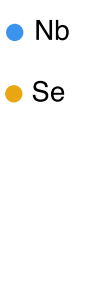

(b)
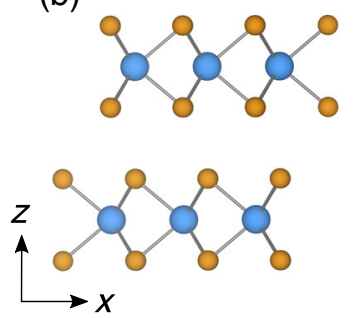

FIG. 1. (a) Crystal structure of bulk $\mathrm{NbSe}_{2}$ illustrating the (a) top view and (b) side view of the structure. The $x, y$, and $z$ axes denote the Cartesian axes.

in agreement with experimental reports of the lattice constants of bulk $\mathrm{NbSe}_{2}$ [40].

The trigonal crystal field splits the $4 d$ states of $\mathrm{Nb}^{4+}$ into three different groups: $d_{z^{2}},\left[d_{x^{2}-y^{2}}, d_{x y}\right]$, and $\left[d_{x z}, d_{y z}\right]$. The bulk band structure of $\mathrm{NbSe}_{2}$, which has been studied extensively [41,42], has three bands that cross the Fermi level. Two of the bands are derived from $\mathrm{Nb} d$ states and the third band is derived from Se $p_{z}$ states. Spin-orbit interaction leads to a mixing of the Se $p$ and $\mathrm{Nb} d$ states along the $\Gamma-K-M$ path of the Brillouin zone, but does not lead to SO splitting. The density of states at the Fermi level $N\left(E_{F}\right)$ is 2.7 states/(eV cell), which leads to a bulk Pauli susceptibility, $\chi_{0}=0.87 \times 10^{-4} \mathrm{emu} / \mathrm{mole}$, which is a factor of $\sim 3.5$ lower than the experimentally reported spin susceptibility of bulk $\mathrm{NbSe}_{2}$ [33], which suggests that spin fluctuations are operative in $\mathrm{NbSe}_{2}$.

For the case of the monolayer, there is one band that crosses the Fermi level several times, leading to three Fermi contours, one contour around the $\Gamma$ point and two contours around $K$ and $K^{\prime}$ (these are related by inversion symmetry). At $\Gamma$, the band character is $\mathrm{Nb} d_{z^{2}}$, with a minor admixture of Se $p_{z}$. As the band progresses toward $K$ or $K^{\prime}$, this leads to a larger admixture of $\mathrm{Nb} d_{x^{2}-y^{2}}$ and $d_{x y}$ orbitals in addition to a minor contribution of Se $p_{x y}$ states. The states at the $K$ and $K^{\prime}$ contours are composed entirely of $\mathrm{Nb}\left[d_{x^{2}-y^{2}}, d_{x y}\right]$ states. In the absence of SO interaction, this band is spin degenerate. When we allow for SO interaction, the lack of a center of inversion in the monolayer leads to SO splitting everywhere except along the $\Gamma-M$ line [cf. Fig. 2(a)], consistent with prior calculations of the band structure of monolayer $\mathrm{NbSe}_{2}$ [43].

To understand why the pseudospin state does not have an in-plane component and why the splitting is small near $\Gamma$, it is instructive to rationalize this splitting from the band structure point of view. If we neglect the minor admixture of Se $p$ states to the bands that cross the Fermi level in monolayer $\mathrm{NbSe}_{2}$, a state at a given wave vector $\mathbf{k}$ can be defined as follows:

$$
|\phi\rangle=\eta\left|d_{x^{2}-y^{2}}\right\rangle+\beta\left|d_{x y}\right\rangle+\gamma\left|d_{z^{2}}\right\rangle
$$
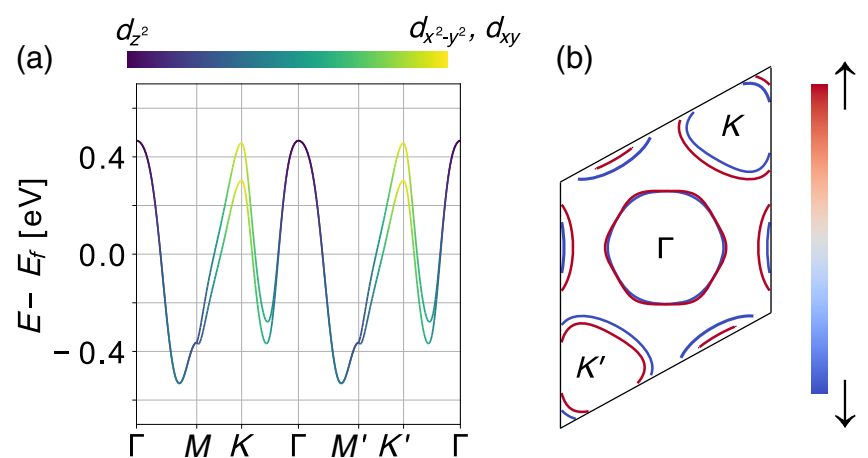

(c)

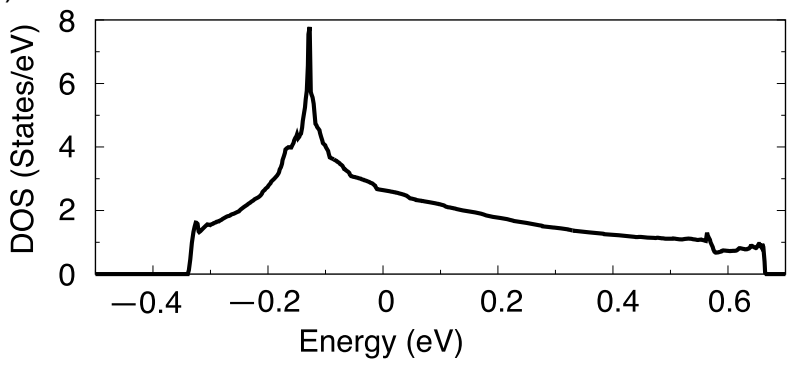

FIG. 2. (a) Band structure of monolayer $\mathrm{NbSe}_{2}$. The color along each band denotes the relative $\mathrm{Nb} d_{z^{2}}, d_{x^{2}-y^{2}}$, and $d_{x y}$ character along the high-symmetry path, according to the color bar above the plot. (b) Cross section of the Fermi surface of monolayer $\mathrm{NbSe}_{2}$. Red denotes bands that have pure $m_{z}=1$ character while blue denotes bands that have pure $m_{z}=-1$ character. (c) Density of states of monolayer $\mathrm{NbSe}_{2}$. All of the calculations include $\mathrm{SO}$ coupling.

where $|\eta|^{2}+|\beta|^{2}+|\gamma|^{2}=1$. Note that $d_{z^{2}}$ corresponds to $|l, m\rangle=|2,0\rangle, \quad d_{x^{2}-y^{2}}$ to $(|2,2\rangle+|2,-2\rangle) \sqrt{2}$, and $d_{x y}$ to $(|2,2\rangle-|2,-2\rangle) / i \sqrt{2}$. Accounting for spin, the Hamiltonian at each $\mathbf{k}$ point is a $(2 \times 2)$ matrix, and, by virtue of the $z /-z$ mirror symmetry, does not include contributions from the $|2, \pm 1\rangle$ orbitals. Thus, the nondiagonal matrix elements $L_{ \pm}$are zero. However, it is easy to show that the diagonal element $L_{z}=2(\eta \operatorname{Im} \beta-\beta \operatorname{Im} \eta)$. One phase can always be selected as real, for instance, $\eta$, then $L_{z}=2 \eta \operatorname{Im} \beta$.

In the centrosymmetric bulk $2 \mathrm{H}-\mathrm{NbSe}_{2}, \beta$ can also be chosen to be real, and there is no SO-induced spin splitting (but there is splitting due to doubling of the unit cell). In the monolayer, $\beta$ is complex everywhere except the $\Gamma-M$ direction, and therefore the diagonal elements of this $(2 \times 2)$ matrix have opposite signs, $\pm \lambda \eta \operatorname{Im} \beta$, where $\lambda$ measures the strength of the SO coupling. Consequently, the splitting is small around the $\Gamma$ pocket (the maximum splitting at this Fermi surface is $\sim 70 \mathrm{meV}$, which occurs where it intersects with the $\Gamma-K$ and $\Gamma-K^{\prime}$ lines), where $|\gamma|^{2} \gg|\eta \beta|$, but sizable $(\sim 150 \mathrm{meV})$ on the $K$ and $K^{\prime}$ contours, where $|\gamma|^{2} \ll|\eta \beta|$. Due to the absence of nondiagonal coupling, the pseudospin-split states are also pure $S_{z}= \pm \frac{1}{2}$ spin states and the direction of the pseudospin flips 
between the $K$ and $K^{\prime}$ valleys as illustrated in Fig. 2(b). As we discuss later, pseudospin states are no longer pure $S_{z}$ states when an external in-plane magnetic field is applied.

\section{MAGNETISM WITHOUT SPIN-ORBIT COUPLING}

To quantitatively address the role of spin fluctuations we calculate the spin susceptibility $\chi$ by considering the effect that a uniform external magnetic field $H$ has on the magnetic moment $m$ in $\mathrm{NbSe}_{2}$, via the Zeeman interaction, where $\chi=\partial m / \partial H$. In practice, one way to simulate the effect of a magnetic field within a first-principles calculation is to apply a constraint on the magnetization and compute the total energy $E$ as a function of the magnetic moment $m$. This "fixed-spin moment" approach allows us to define $\chi$ as $\chi=\left(\partial^{2} E / \partial m^{2}\right)^{-1}$. Similarly, noncollinear FSM calculations that include SO interaction let us determine the change in total energy and in turn $\chi$ for directions parallel to the $c$ axis $(\langle 001\rangle)$ and perpendicular to the $c$ axis $(\langle 100\rangle)$ of $\mathrm{NbSe}_{2}$.

The collinear and noncollinear (along $\langle 001\rangle$ and $\langle 100\rangle$ ) FSM calculations result in the same qualitative trends; the total energy increases monotonically with respect to the total energy of the nonmagnetic state as a function of increasing magnetic moment. As an example, we illustrate the results of our collinear FSM calculations for bulk and monolayer $\mathrm{NbSe}_{2}$ in Fig. 3. If we express the expansion of the DFT total energy as

$E(m)=a_{0}+a_{1} m^{2}+a_{2} m^{4}+a_{3} m^{6}+a_{4} m^{8}+\cdots$,

we can determine the static spin susceptibility $\chi$ for low values of $m$ by using the coefficient $a_{1}$ obtained by fitting the data in Fig. 3 to Eq. (2). The results are summarized in Table I.

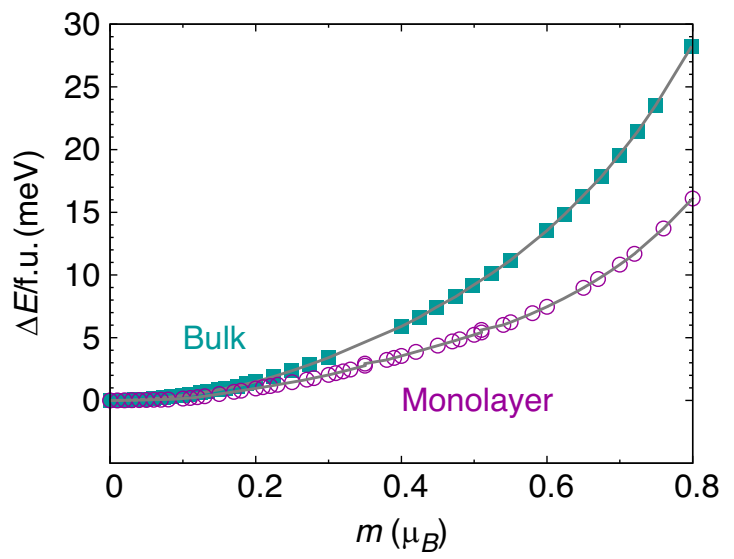

FIG. 3. Change in total energy per $\mathrm{NbSe}_{2}$ formula unit (f.u.) of the bulk (square) and monolayer (circle) structures with respect to the nonmagnetic state as a function of magnetic moment obtained from collinear fixed-spin moment calculations. The gray solid lines are a fit to Eq. (2).
Based on our FSM calculations, we can draw the following conclusions. First, DFT repoduces the experimentally observed bulk susceptibility reasonably well, only slightly overestimating it compared to the experimental value. This overestimation in the calculated value of $\chi$ is known to occur in itinerant metals close to a magnetic instability, and is due to a fluctuational reduction of the mean-field DFT moment [44,45]. Applying Moriya's theory [44] to $\mathrm{NbSe}_{2}$, we can estimate the average magnitude of spin fluctuations as $\xi \sim 0.28 \mu_{B}$. To put this into context, the average magnitude of spin fluctuations in palladium (Pd), a known superparamagnet (which at some point was considered a candidate for triplet superconductivity [35]) was calculated (in local density approximation, as opposed to our generalized gradient approximation calculation) to be $\xi \sim 0.15 \mu_{B}$ [45].

This is also consistent with our disordered local moment (DLM) calculations, where the energy cost of creating a local spin fluctuation with an amplitude of $\sim 0.2 \mu_{B}$ is nearly twice higher in $\mathrm{Pd}$ than in $\mathrm{NbSe}_{2}$. This does not mean that $\mathrm{NbSe}_{2}$ is closer to ferromagnetism compared to Pd. The molar spin susceptibility of $\mathrm{NbSe}_{2}$ is a factor of 2 lower compared to Pd. However, it does mean spin fluctuations in $\mathrm{NbSe}_{2}$ are soft over a large part of the Brillouin zone. Furthermore, it is important to note the susceptibility of the monolayer structure is $\sim 50 \%$ larger than that of the bulk, which indicates that spin fluctuations are stronger in a monolayer. Indeed, this is consistent with monolayer $\mathrm{NbSe}_{2}$ having a lower superconducting transition temperature compared to bulk $\mathrm{NbSe}_{2}$ [15].

To verify that $\mathrm{NbSe}_{2}$ is indeed close to a ferromagnetic instability, we also calculated the exchange coupling between fluctuating moments within the DLM formalism (the calculation details are similar to methods used in Ref. [46]) for bulk $\mathrm{NbSe}_{2}$. The exchange coupling is largely dominated by the nearest-neighbor coupling, which we find to be ferromagnetic. In order to transform the exchange interactions into reciprocal space $J_{0}(\mathbf{q})$, we have defined $\chi_{\mathrm{RPA}}(\mathbf{q})=c_{0} /\left[1-c_{0} J_{0}(\mathbf{q})\right]$, where $c_{0}$ is a constant of the

TABLE I. Spin susceptibility of the bulk and monolayer structures obtained from collinear and noncollinear calculations with the spin quantization axis parallel to the $c$ axis, $\langle 001\rangle$, and the spin quantization axis along the $x$ direction, $\langle 100\rangle$. The calculated susceptibility along $\langle 100\rangle,\langle 010\rangle$, and $\langle 110\rangle$ are equivalent. The experimental value of $\chi$, obtained from Ref. [33], is for a magnetic field applied parallel to the $c$ axis $(\langle 001\rangle)$. The experimental value of $\chi$ for monolayer $\mathrm{NbSe}_{2}$ to our knowledge has not been reported yet.

\begin{tabular}{lcc}
\hline \hline Spin susceptibility & Bulk & $\begin{array}{c}\text { Monolayer } \\
{\left[10^{-4} \mathrm{emu} / \mathrm{mole}\right]}\end{array}$ \\
\hline Collinear & 4.28 & 6.81 \\
$\langle 001\rangle$ & 4.20 & 7.29 \\
$\langle 100\rangle$ & 4.23 & 7.40 \\
Experiment & $\sim 3$ & $\cdots$ \\
\hline \hline
\end{tabular}




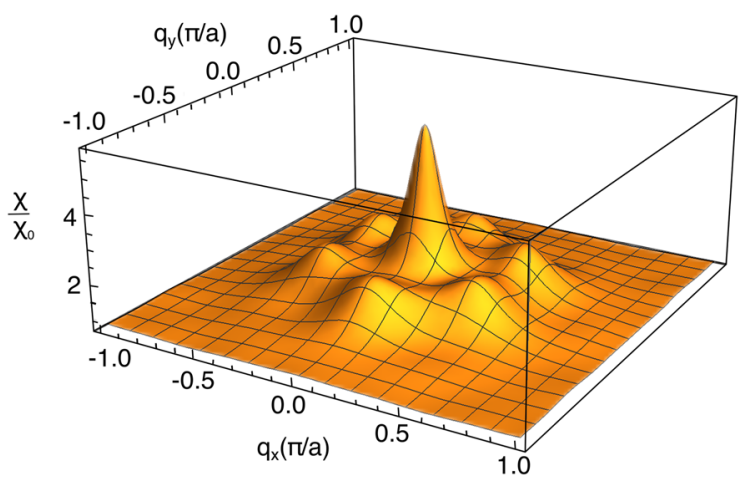

FIG. 4. q-dependent Stoner renormalization factor of bulk $\mathrm{NbSe}_{2}$, obtained from disordered local moment calculations.

order of $N(0)$. In Fig. 4 we plot the renormalization factor $1 /\left[1-c_{0} J(\mathbf{q})\right]$, using $c_{0}=3.57 \mathrm{eV}^{-1}$, which was chosen so as to have the renormalization at $\mathbf{q}=0$ be approximately consistent with a renormalization factor of 4.9. The peak near $\mathbf{q}=0$ in Fig. 4 indicates that the system is close to a ferromagnetic instability.

The DLM calculations allow us to determine the $\mathbf{q}$ dependence of $\chi$ and offers qualitative insight into the magnitude of $\chi$. For accurate quantitative values of $\chi$, see Table I, which are obtained using FSM calculations.

\section{MAGNETISM WITH SPIN-ORBIT COUPLING}

Having demonstrated that bulk and monolayer $\mathrm{NbSe}_{2}$ are indeed close to a ferromagnetic instability, we now focus on the effect of SO coupling, and specifically on the response to an external magnetic field applied parallel to the $c$ axis and perpendicular to the $c$ axis. First, it is evident the values of $\chi$ reported in Table I from our noncollinear calculations are isotropic along $\langle 001\rangle$ and $\langle 100\rangle$, within a few percent, for both the bulk and monolayer structures, as opposed to the susceptibility in the superconducting state.

To understand this, let us analyze how the bands that cross the Fermi level evolve as a function of the magnitude and direction of an applied magnetic field. In the absence of SO interaction, the states at $\Gamma, K$, and $K^{\prime}$ are degenerate. The Zeeman interaction, regardless of the direction of the field, splits the bands by approximately the same magnitude, $\pm H$, where $H$ is the Stoner-enhanced external field (we have absorbed the Bohr magneton in the units of $H$ ). Indeed, in our calculations, the splitting at $\Gamma, K$, and $K^{\prime}$ increases linearly and by approximately the same amount as a function of increasing magnetic moment. The magnitude of the Fermi surface splitting in reciprocal space is $\delta k_{F}(\mathbf{k})=2 H / v_{F}(\mathbf{k})$. The area between the spin-split contours will determine the total magnetization for a given magnitude of the magnetic field, and will be $2 N_{\uparrow} H=$ $N_{\uparrow \downarrow} H$, where $N_{\uparrow}$ is the total number of states with pseudospins along $\hat{z}$ and $N_{\uparrow \downarrow}$ is the total number of states with pseudospins along $\hat{z}$ and $-\hat{z}$.
We now define the following generic Hamiltonian with $\mathrm{SO}$ interaction for a given point on the Fermi surface of monolayer $\mathrm{NbSe}_{2}$ subject to an external magnetic field $H_{z}$ along $\hat{z}$ (parallel to the $c$ axis), where the spin-quantization axis is along $\hat{z}$ :

$$
\mathfrak{H}(\mathbf{k})=\left(\begin{array}{cc}
\varepsilon_{\mathbf{k}} \pm \lambda_{\mathbf{k}}+H_{z} & 0 \\
0 & \varepsilon_{\mathbf{k}} \mp \lambda_{\mathbf{k}}-H_{z}
\end{array}\right) .
$$

Based on Eq. (1), $\lambda_{\mathbf{k}}=\lambda \eta_{\mathbf{k}} \operatorname{Im} \beta_{\mathbf{k}}$, and the matrix is in the $S_{z}$ spin space. Inversion in the momentum space changes the upper sign to the lower sign for $\pm \lambda$ and $\mp \lambda$. The splitting between the Fermi contours for a given pseudospin along $+\hat{z}$ will increase by approximately the same magnitude as the Fermi contours of the majority spin channel in the case of the collinear calculations. The splitting of the Fermi contours for pseudospins along $-\hat{z}$ will decrease by the same magnitude. For instance, if the Fermi contour splitting around $\mathrm{K}$ increases by $2 H_{z}$, the splitting around $K^{\prime}$ will decrease by the same amount. Hence, the total magnetization that is induced in terms of the population of pseudospins, to lowest order in $\lambda$, will be exactly the same as determined by our collinear calculations.

In the noncollinear DFT calculations, a pseudospin state is formally a combination of both spins, so we introduce an effective $g$ factor, which describes the difference between the pure spin susceptibility and the "pseudospin" susceptibility. For a magnetic field along $\hat{z}$, the $g$ factor is exactly 2 , so we expect $\chi$ along $\langle 001\rangle$ to be very similar to $\chi$ obtained from collinear calculations-which is consistent with our calculations in Table I. The splitting of the states at $\Gamma, K$, and $K^{\prime}$ for magnetic moments along $\hat{z}$ is illustrated in Fig. 5(a) and they indeed change linearly with respect to the magnetic moment.

For an in-plane magnetic field along $\hat{x}$ (perpendicular to the $c$ axis), $H_{x}$, the Zeeman interaction, $S_{x} H_{x}=$ $\left(S_{+}+S_{-}\right) H_{x} / 2$, couples to the off-diagonal components of the spin-orbit Hamiltonian as follows:

$$
\mathfrak{H}(\mathbf{k})=\left(\begin{array}{cc}
\varepsilon_{\mathbf{k}} \pm \lambda_{\mathbf{k}} & \pm H_{x} / 2 \\
\mp H_{x} / 2 & \varepsilon_{\mathbf{k}} \mp \lambda_{\mathbf{k}}
\end{array}\right) .
$$

To linear order in $H_{x}$, the splitting between the Fermi contours at $K$ and $K^{\prime}$ will not change. However, the wave functions change, and thus the effective $g$ factor will deviate linearly from 2 . For example, applying standard perturbation theory to the pseudospin $|+\rangle$ states gives

$$
\begin{aligned}
|+\rangle & =|\uparrow\rangle \pm \frac{H_{x} / 2}{2 \lambda_{\mathbf{k}}}|\downarrow\rangle, \\
\left\langle+\left|\frac{\sigma_{+}+\sigma_{-}}{2}\right|+\right\rangle & = \pm \frac{H_{x} / 2}{\lambda_{\mathbf{k}}} .
\end{aligned}
$$

Pseudospin $|-\rangle$ states will acquire the opposite magnetization, and their $g$ factor will be reduced by the same amount. We now observe that the total pseudomoment 

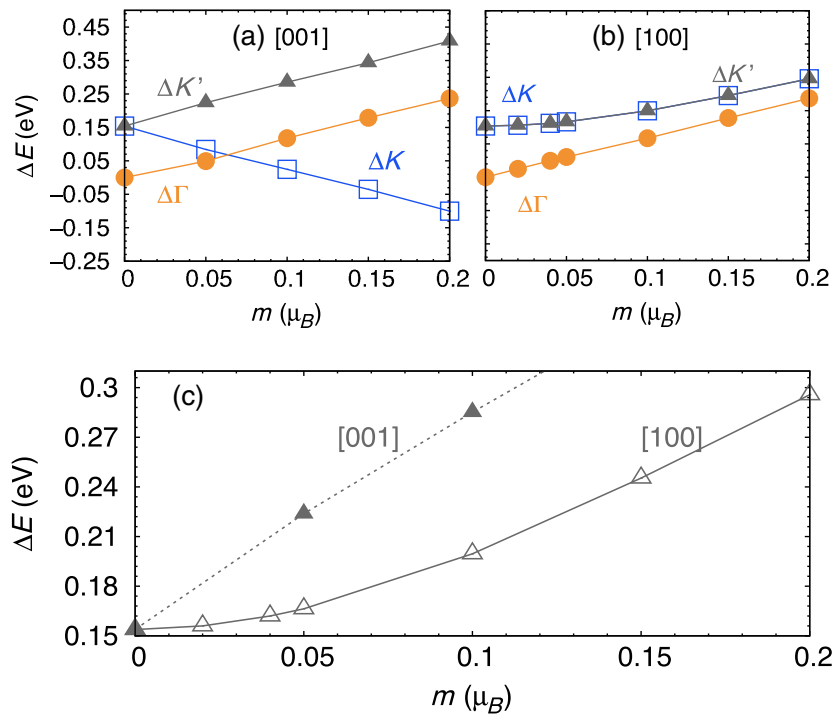

FIG. 5. Splitting at $\Gamma$ (circle), $K$ (square), and $K^{\prime}$ (triangle) obtained with noncollinear calculations as a function of the magnetic moment on $\mathrm{Nb}$ for magnetic moments (a) parallel to the $c$ axis, $\langle 001\rangle$, and (b) perpendicular to the $c$ axis, $\langle 100\rangle$, for monolayer $\mathrm{NbSe}_{2}$. The magnitude of the splitting for magnetic moments along $\langle 010\rangle$ are similar to the results along $\langle 100\rangle$ illustrated in (b). The solid line in (a) and (b) for each plot serves as a guide to the eye. (c) Splitting at $K^{\prime}$ for magnetic moments on $\mathrm{Nb}$ parallel to the $c$ axis ([001], dotted line) illustrating the linear dependence on $m$ and magnetic moments perpendicular to the $c$ axis ([100], solid line) illustrating the quadratic dependence on $m$. The results are the same at $K$ if one considers the magnitude of the change in $\Delta E$ versus $m$.

around the $K$ point will be proportional to the area between the split concentric Fermi contours, $\pm N_{K} \lambda_{K}$, where $N_{K}$ is the density of states for this contour at $K$ and $\lambda_{K}$ is the average splitting at this contour. Around the $K^{\prime}$ contour, the area between the split concentric Fermi contours is $\mp N_{K} \lambda_{K}$, and this total pseudomoment does not depend on $H_{x}$. Multiplying it by the difference in the $g$ factors of Eq. (4), which deviate from 2 by the same amount, but in the opposite directions, we get a spin susceptibility of $\chi_{\langle 100\rangle} \approx N_{\text {tot }}=\chi_{\text {Pauli }}$, where $N_{\text {tot }}$ is the total density of states. Thus, no anisotropy in the spin susceptibility appears in the lowest order of the SO coupling. DFT calculations fully conform with this description: the splitting of the one-electron energies at $K$ and $K^{\prime}$ is quadratic with respect to magnetic moments oriented along $\hat{x}$ [cf. Fig. 5(b)].

Within our considerations of the Zeeman interaction, $H$ is the total magnetic field, which includes the Stoner renormalization. Within DFT, the RPA is exact, since one can write the total DFT exchange-correlation energy $E_{\mathrm{xc}}$ in an external magnetic field as $[47,48]$

$$
E_{\mathrm{xc}}=\frac{m^{2}}{4}\left(\frac{1}{N_{\uparrow}}-I\right),
$$

where $I=\delta^{2} E_{\mathrm{xc}} / d m^{2}$ is the DFT Stoner factor, which in the DFT language combines the diagonal (Hubbard $U$ ) and offdiagonal (Hund's $J$ ) interactions. Indeed,

$$
\begin{aligned}
\chi_{\mathrm{DFT}} & =\chi_{0} /\left(1-\chi_{0} I\right), \\
H & =H_{\mathrm{ext}} /\left(1-\chi_{0} I\right),
\end{aligned}
$$

where $\chi_{0}$ is the bare Pauli susceptibility.

\section{MAGNETISM AND SUPERCONDUCTIVITY}

Within this framework, it is especially easy to address the effect of superconductivity on the spin susceptibility. Indeed, the opening of the superconducting gap $\Delta$ only affects states that are close to the Fermi surface. Since the spin susceptibility parallel to the $c$ axis, $\chi_{\langle 001\rangle}$, is determined entirely by the shift of the Fermi contours as a function of an increasing magnetic field [Fig. 5(a)], the spin susceptibility is suppressed by superconductivity in exactly the same way as without SO coupling. In contrast, the spin susceptibility perpendicular to the $c$ axis, $\chi_{\langle 100\rangle}$, as we just saw, is defined by the states removed from the Fermi level by $\sim \lambda_{\mathbf{k}} \gg \Delta$, and as a result is not affected by $\Delta$. Thus, the thermodynamic critical field, $H_{C 0}$, which is determined by the free energies in the normal and superconducting state as

$$
F_{n}-F_{s} \sim \Delta^{2} N_{\uparrow \downarrow}(0) / 2=\left(\chi_{n}-\chi_{s}\right) H_{C 0}^{2} / 2,
$$

behaves conventionally for magnetic fields parallel to the $c$ axis, but is essentially infinite for magnetic fields perpendicular to the $c$ axis.

However, if one examines the SO splitting for the Fermi contour around the $\Gamma$ point, we find that it is nodal along the $\Gamma-M$ and the $\Gamma-M^{\prime}$ line, which makes $H_{C 0}$ finite, but greatly enhanced for magnetic fields perpendicular to the $c$ axis. Figure 2(b) illustrates the calculated splitting due to SO coupling along the $\Gamma$ contour is finite and has nodes along all $\Gamma-M$ and $\Gamma-M^{\prime}$ directions. The magnitude of this splitting is low, but, along the antinodal line $\Gamma-K$, the maximum splitting is still larger than the superconducting gap. In the Appendix $\mathrm{C}$ we derive an analytical expression, which generalizes the considerations presented above onto a SO-coupled nodal case of the $\Gamma$-centered Fermi contour.

\section{SINGLET AND TRIPLET SUPERCONDUCTIVITY: DFT POINT OF VIEW}

We now use the above considerations to determine the symmetry of possible pairing interactions for monolayer $\mathrm{NbSe}_{2}$. The fact that $\mathrm{NbSe}_{2}$ lacks inversion symmetry formally allows for parity mixing, but it has been argued [18] that the triplet component must be vanishingly small. As we discuss below, this is not necessarily true. Strong spin fluctuations, which we have demonstrated to be operative in $\mathrm{NbSe}_{2}$, and/or a particular structure of the 
electron-phonon coupling have the ability to generate a sizable triplet component.

In the spirit of band theory, we consider the one-electron Hamiltonian to be fully diagonalized before we consider superconducting pairing. First, we only consider the $K$ and $K^{\prime}$ contours. The Cooper pairs at these contours are composed of states that reside on either the inner or outer contours at $K$ and $K^{\prime}$. We assume that the outer contour around $K$ has spin-up states, and the inner contour has spindown states, which we denote as $[|K, o, \uparrow\rangle,|K, i, \downarrow\rangle]$. The contours at $K^{\prime}$ are degenerate in energy with the contours at $K$ (the states at $K$ and $K^{\prime}$ are related by time-reversal symmetry) and are given by $\left[\left|K^{\prime}, o, \downarrow\right\rangle,\left|K^{\prime}, i, \uparrow\right\rangle\right]$. No other combinations are allowed. Schematically, these four contours can be represented as two pairs of concentric rings as depicted in Fig. 6. In this basis, the anomalous averages that appear in the problem are

$$
\begin{aligned}
d_{o, \mathbf{k}} & =\overline{|K, o, \uparrow\rangle\left|K^{\prime}, o, \downarrow\right\rangle}, \\
d_{i, \mathbf{k}} & =-\overline{|K, i, \downarrow\rangle\left|K^{\prime}, i, \uparrow\right\rangle} .
\end{aligned}
$$

Note that we introduced a minus sign in Eq. (8) for $d_{i, \mathbf{k}}$; this is to ensure that a usual spin-singlet state is given by $d_{i, \mathbf{k}}=d_{o, \mathbf{k}}$ and that the interactions we discuss later reduce to the usual interactions when no spin-orbit coupling is included. Indeed, one is free to define the relative phase between the superconducting order parameters at different momenta, without changing the superconducting state or any observables. Usually there is one logical choice and it is universally used. For the case of $\mathrm{NbSe}_{2}$ it is important to note that the relative sign between the order parameter at $K$ and $K^{\prime}$ is not uniquely defined. Hence, one needs to be careful when defining the phase convention for a given

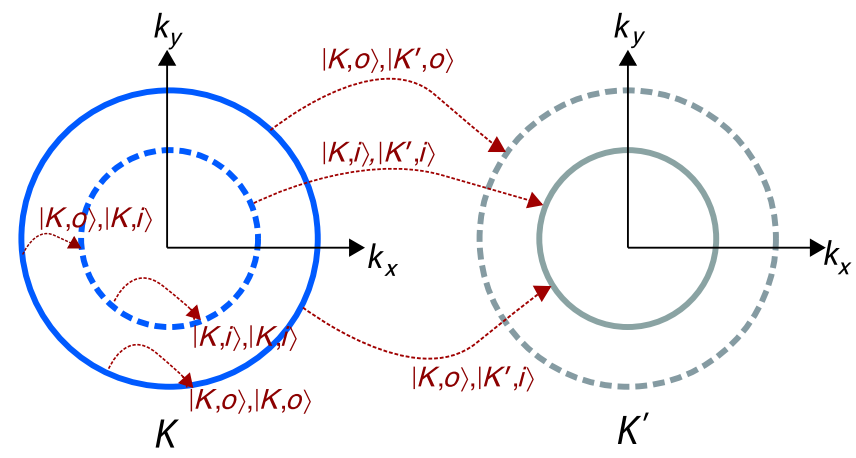

FIG. 6. Schematic illustration of the inner and outer contours of the Fermi surface around the $K$ and $K^{\prime}$ points that cross the Fermi level [cf. Fig. 2(b)]. Solid circles represent pseudospin $|\uparrow\rangle$ states and dotted circles represent pseudospin $|\downarrow\rangle$ states. The possible pairing interactions due to phonons or spin fluctuations between the pseudospin states are denoted with red dotted arrows. The relative signs of these interactions are summarized in Table II. Subscript $o$ refers to the outer contour and the subscript $i$ refers to the inner contour at a given valley. pairing interaction, as it can have an instrumental impact as has been shown for other materials [49].

Since a singlet pair is defined as $(|\uparrow \downarrow\rangle-|\downarrow \uparrow\rangle) / \sqrt{2}$ and the triplet pair is defined as $(|\uparrow \downarrow\rangle+|\downarrow \uparrow\rangle) / \sqrt{2}$, the order parameter on the outer contour $\Delta_{o}$, derived from the anomalous average $d_{o}$, is $\Delta_{o}=\left(\Delta_{S}+\Delta_{T}\right) / \sqrt{2}$, while the order parameter on the inner contour $\Delta_{i}$ is $\Delta_{i}=$ $\left(\Delta_{S}-\Delta_{T}\right) / \sqrt{2}$. Within this definition, $\Delta_{S}$ is the order parameter for a singlet pair and $\Delta_{T}$ is the order parameter for a triplet pair. Note that the symbols $\Delta_{i}, \Delta_{o}, \Delta_{S}$, and $\Delta_{T}$ that are used in this discussion always refer to the superconducting order parameter which can be different from the superconducting excitation gap $\Delta$. This picture implies four types of pairing interactions, which corresponds to the following scattering processes of Cooper pairs: $d_{o} \Leftrightarrow d_{o}$, $d_{i} \Leftrightarrow d_{i}, d_{o} \Leftrightarrow d_{i}, d_{i} \Leftrightarrow d_{o}$. If $\Delta_{o}=\Delta_{i}$, then in most (albeit not necessarily all) experiments the triplet component cancels out. For example, such a situation can arise following the considerations of Shaffer et al. [18], where they take the intraband scattering within the same valley, $d_{o, K} \Leftrightarrow d_{o, K}$ or $d_{i, K} \Leftrightarrow d_{i, K}$, to be the same (denoted as $g_{2}$ in Ref. [18]), which differs from their consideration of intraband scattering between the $K$ and $K^{\prime}$ valley, $d_{o, K} \Leftrightarrow d_{i, K^{\prime}}$, $d_{i, K} \Leftrightarrow d_{o, K^{\prime}}$ (denoted as $g_{3}$ in Ref. [18]).

If the pairing is due to phonons, then the matrix element for intraband pairing interactions $g_{o o}^{p}$ (or $g_{i i}^{p}$ ) between the momenta $\mathbf{k}$ and $\mathbf{p}$ is defined as

$$
\begin{aligned}
\left\langle d_{o, \mathbf{k}}|v| d_{o, \mathbf{p}}\right\rangle & =\langle\mathbf{k}, o, \uparrow|\langle-\mathbf{k}, o, \downarrow|v| \mathbf{p}, o, \uparrow\rangle|-\mathbf{p}, o, \downarrow\rangle \\
& =\langle\mathbf{k}, o, \uparrow|g| \mathbf{p}, o, \uparrow\rangle\langle-\mathbf{k}, o, \downarrow|g|-\mathbf{p}, o, \downarrow\rangle \\
& =g_{o o}^{p},
\end{aligned}
$$

where the phonon Green's function is included in $g$. The nondiagonal electron-phonon matrix element that couples the outer and inner contours $g_{o i}^{p}$ is defined as

$$
\begin{aligned}
\left\langle d_{o, \mathbf{k}}|v| d_{i, \mathbf{p}}\right\rangle & =\langle\mathbf{k}, o, \uparrow|\langle-\mathbf{k}, o, \downarrow|v| \mathbf{p}, i, \downarrow\rangle|-\mathbf{p}, i, \uparrow\rangle \\
& =\langle\mathbf{k}, o, \uparrow|g|-\mathbf{p}, i, \uparrow\rangle\langle-\mathbf{k}, o, \downarrow|g| \mathbf{p}, o, \downarrow\rangle \\
& =g_{o i}^{p} .
\end{aligned}
$$

Since scattering by phonons does not flip spin, electronphonon coupling is only relevant for $|K, \nu\rangle \Leftrightarrow\left|K, \nu^{\prime}\right\rangle$ scattering if $\nu=\nu^{\prime}$ or for $|K, \nu\rangle \Leftrightarrow\left|K^{\prime}, \nu^{\prime}\right\rangle$ if $\nu \neq \nu^{\prime}$. The indices $\nu$ and $\nu^{\prime}$ can be either $o$ or $i$ to denote a state on an outer $(o)$ or inner $(i)$ contour.

On the other hand, ferromagnetic spin fluctuations, even though they can only couple states within the same valley, can work within both the inter- and intraband channels. To determine the relative sign and strength of these interactions, we define the amplitude of a spin fluctuation as $\mathbf{S}$, and to a reasonable approximation their correlators can be assumed to be spin-rotationally invariant: $\left\langle S_{\mathbf{k}}^{z} S_{\mathbf{p}}^{z}\right\rangle=$ $\left\langle S_{\mathbf{k}}^{x} S_{\mathbf{p}}^{x}\right\rangle=\left\langle S_{\mathbf{k}}^{y} S_{\mathbf{p}}^{y}\right\rangle=\left\langle S_{\mathbf{k}}^{+} S_{\mathbf{p}}^{-}\right\rangle / 2$, leading to an isotropic spin 
susceptibility $\chi$. Within this definition, we can now describe the interaction of an electronic state that crosses the Fermi level with a fluctuating spin moment as $\sigma \cdot \mathbf{S}=\sigma^{z} S^{z}+$ $\left(\sigma^{+} S^{-}+\sigma^{-} S^{+}\right) / 2$. Hence, just as we did for the intraband electron-phonon interaction [Eq. (9)], we can write down the following expression for the intraband interaction due to spin fluctuations $g_{o o}^{s}\left(\right.$ or $\left.g_{i i}^{s}\right)$ :

$$
\begin{aligned}
\left\langle d_{o, \mathbf{k}}|\langle\mathbf{S} \otimes \mathbf{S}\rangle| d_{o, \mathbf{p}}\right\rangle= & \left\langle\mathbf{k}, o, \uparrow\left|\sigma^{z} S^{z}\right| \mathbf{p}, o, \uparrow\right\rangle \\
& \times\left\langle-\mathbf{k}, o, \downarrow\left|\sigma^{z} S^{z}\right|-\mathbf{p}, o, \downarrow\right\rangle, \\
g_{o o}^{s}= & -\frac{1}{4}\langle\mathbf{k}, o \mid \mathbf{p}, o\rangle \chi(\mathbf{k}-\mathbf{p}) .
\end{aligned}
$$

Note that the sign of $g_{o o}^{s}$ is negative, indicating intraband spin-fluctuation mediated interactions are repulsive, as would occur in a singlet channel. We also define an expression for spin-fluctuation mediated interactions that couple the outer and inner contours $g_{o i}^{s}$ as

$$
\begin{aligned}
\left\langle d_{o, \mathbf{k}}|\langle\mathbf{S} \otimes \mathbf{S}\rangle| d_{i, \mathbf{p}}\right\rangle= & -\left[\left\langle\mathbf{k}, o, \uparrow\left|\sigma^{+} S^{-}\right| \mathbf{p}, i, \downarrow\right\rangle\right. \\
& \left.\times\left\langle-\mathbf{k}, o, \downarrow\left|\sigma^{-} S^{+}\right|-\mathbf{p}, i, \uparrow\right\rangle\right] / 4, \\
g_{o i}^{s}= & -\frac{1}{2}\langle\mathbf{k}, o \mid \mathbf{p}, i\rangle \chi(\mathbf{k}-\mathbf{p}),
\end{aligned}
$$

where the minus sign in this matrix element appears because of our phase convention for $\Delta_{i}$. Note that the prefactor $g_{o i}^{s}$ is a factor of 2 larger than $g_{o o}^{s}$.

Hence, intraband and interband interactions around a fixed valley $\left(K\right.$ or $\left.K^{\prime}\right)$ have the same sign as expected for singlet pairs. However, they also have distinct prefactors, which is as if the standard singlet rotational factor of 3 has distributed itself in a ratio of $2: 1$ between the interband and intraband contributions within the SO-split bands. This combination of pairing interactions due to phonons and spin fluctuations is summarized in Table II. We have

TABLE II. Sign of the pairing interaction that involves either phonons $g^{p}$ or ferromagnetic spin fluctuations $g^{s}$ between two states on the outer and/or inner contour at $K$ and $K^{\prime}$. The states involved in pairing are denoted as $|\mathbf{k}, \nu\rangle$, where $\mathbf{k}$ is a state either at $K$ or $K^{\prime}$ and $\nu$ can take on an index $o$ or $i$ to denote a state on the outer $(o)$ or inner $(i)$ contour. The pseudospin ( $\uparrow$ or $\downarrow$ ) associated with states on each contour is depicted schematically in Fig. 6. Attractive pairing interactions are positive and repulsive interactions are negative.

\begin{tabular}{lcc}
\hline \hline Pair & $g^{p}$ & $g^{s}$ \\
\hline$|K, o\rangle,|K, o\rangle$ & $g_{o o}^{p}>0$ & $g_{o o}^{s}<0$ \\
$|K, i\rangle,|K, i\rangle$ & $g_{i i}^{p}>0$ & $g_{i i}^{s}<0$ \\
$|K, o\rangle,\left|K^{\prime}, o\right\rangle$ & 0 & $\approx 0$ \\
$|K, i\rangle,\left|K^{\prime}, i\right\rangle$ & 0 & $\approx 0$ \\
$|K, o\rangle,|K, i\rangle$ & 0 & $g_{o i}^{s}<0$ \\
$|K, o\rangle,\left|K^{\prime}, i\right\rangle$ & $g_{o i}^{p}>0$ & $\approx 0$ \\
\hline \hline
\end{tabular}

provided an alternative and more complete derivation of these interactions in Appendix B.

The line of reasoning that leads to $\Delta_{o}=\Delta_{i}$ is based on the fact that the bands in question are two dimensional and nearly parabolic, so their DOS is essentially the same (which DFT calculations confirm), while the direction of the spins is antialigned. If the strength of the pairing interaction is similar, this hypothesis of $\Delta_{o}=\Delta_{i}$ is confirmed, and the net superconducting order parameter exhibits a singlet character (see also Appendix B). Note that while the difference in the DOS of the outer and inner contours is vanishingly small, the $k_{F}$ splitting is definitely not negligible in $\mathrm{NbSe}_{2}$. In monolayers of other TMDs, such as $\mathrm{TaS}_{2}$, where Ising superconductivity has been observed, the $k_{F}$ splitting is larger than $\mathrm{NbSe}_{2}$ [16]. The implications of this large splitting of $k_{F}$ on the strength of the pairing interaction on either contour is currently not known. Thus, in the following we ask; does the strength of the pairing interaction have to be similar on the outer and inner contours?

First, we discuss intraband interactions. They have a pairing component due to phonons that is determined by the Eliashberg function, $\alpha^{2} F(\mathbf{q}, \omega)$, which will have a characteristic momentum $\mathbf{q}$ and energy $\omega$ dependence, and a pair-breaking component due to ferromagnetic spin fluctuations, which is determined by the $\mathbf{q}$-dependent spin susceptibility $\chi(\mathbf{q}, \omega)$. The $q$-dependent spin susceptibility is sharply peaked at $q \sim 0$ (Fig. 4). The $q$ dependence of the electron-phonon coupling likely has a non-negligible q dependence as well.

If the intraband interaction due to phonons is the same for states on the outer and inner contours, $g_{o o}^{p}=g_{i i}^{p}$ (an approximation adopted within Ref. [18]), then the ratio of the superconducting gap values, $\left|\Delta_{o}\right| /\left|\Delta_{i}\right|$, is inversely proportional to the square root of the ratio of the density of states [50] of the outer and inner contour, $\sqrt{N_{o} / N_{i}}$, which is, as we know, essentially 1 . The phase, however, depends on the net sign of the interaction: if $\left|g_{o i}^{p}\right|>\left|g_{o i}^{s}\right|$, the phase is the same, and the net order parameter is essentially singlet. However, if $\left|g_{o i}^{p}\right|<\left|g_{o i}^{s}\right|$, which is feasible, the order parameter is net triplet.

Let us now discuss the potential for parity mixing due to a given structure of the intraband coupling. We assume that the net intraband interaction due to electron-phonon $g^{p}(\mathbf{q})$ and spin-fluctuation mediated interactions $g^{s}(\mathbf{q})$ is peaked at small $q$. To be specific, we take this net interaction to have a Lorentzian dependence on $\mathbf{q}$ (it may as well have a sharp minimum at $\mathbf{q}=0$, or have some other comparable structure within momentum space):

$$
g\left(\mathbf{q}=\mathbf{k}-\mathbf{k}^{\prime}\right) \propto \xi^{2} /\left(q^{2}+\xi^{2}\right)
$$

The net intraband coupling constants, $g_{o o}$ and $g_{i i}$, can be obtained by averaging Eq. (13) over all $k$ and $k^{\prime}$ on a circular Fermi contour with a radius $k_{F}$. If we now consider how $g(\mathbf{q})$ changes if $k_{F}$ changes by $\delta k_{F}$, where $\delta k_{F}$ is the 
SO-coupling induced splitting of the Fermi contours, we find

$$
\frac{g_{i i}-g_{o o}}{g_{i i}+g_{o o}}=\frac{\delta k_{F} / k_{F}}{1+\left(\xi / 2 k_{F}\right)^{2}} .
$$

Unlike the DOS, which for a parabolic two-dimensional band does not depend on $k_{F}$, this expression for the net pairing interaction due to intraband interactions depends linearly on $\delta k_{F}$ and thus on the magnitude of the SO coupling. If $\xi \gg 2 k_{F}$, it vanishes, but if $\xi \sim 2 k_{F}$, it leads to a non-negligible correction. Note that in $\mathrm{NbSe}_{2}$, $\delta k_{F} / k_{F} \sim 1 / 3$, and in $\mathrm{TaS}_{2}, \delta k_{F} / k_{F} \sim 1 / 2$. While momentumresolved calculations of the electron-phonon coupling in monolayer $\mathrm{NbSe}_{2}$ are under way [51], the qualitative considerations we have presented above challenge the current notion that the superconducting order parameter in monolayer $\mathrm{NbSe}_{2}$ is purely singlet, and demonstrate the order parameter can indeed host a measurable admixture of triplet character. Furthermore, our consideration of possible pairing interactions in Table II makes it evident that due to the strong spin-orbit interaction of $\mathrm{NbSe}_{2}$, equal-spin triplet state is not possible at zero magnetic field.

Finally, while this is not the main subject of our paper, we briefly comment on the ramifications and plausible experimental probes of the singlet-triplet mixing of the order parameter of monolayer $\mathrm{NbSe}_{2}$. Indeed, a number of recent studies have alluded to the possibility of singlettriplet mixing of the order parameter in monolayer $\mathrm{NbSe}_{2}$ $[17,21,26]$ by invoking extrinsic mechanisms such as impurities and strain. The discussion we have presented above suggests this parity mixing of the order parameter can have an intrinsic origin depending on the interplay between momentum-dependent phonon and spinfluctuation induced couplings. Experiments that attempt to elucidate this parity mixing need to access the paritydependent coherence factors. One possibility is quasiparticle interference, where the main challenge is to separate the intraband $(o-o$ and $i-i)$ scattering from the interband scattering processes. Magneto-optical spectroscopy using microwaves in the deep infrared region of the spectrum is another potential experimental probe. Finally, in the spirit of Ref. [26], one expects that impurities may affect superconductivity differently, depending on the parity. All of these probes require quantitative theories that are beyond the scope of this present paper. Our primary goal was to demonstrate that mixed parity Ising superconductivity is possible in the transition metal dichalcogenides, and we hope this will encourage further theoretical and experimental research into its manifestation.

\section{CONCLUSIONS}

We have developed a formalism that adapts the model theory for Ising superconductivity into first-principles DFT calculations. We demonstrated that bulk and monolayer
$\mathrm{NbSe}_{2}$ are close to a magnetic instability, and spinfluctuation induced interactions cannot be neglected when addressing superconductivity in $\mathrm{NbSe}_{2}$. Finally, we outlined two parametrically admissible situations where superconductivity in monolayer $\mathrm{NbSe}_{2}$ may be partially triplet or even predominantly triplet without invoking an external magnetic field or exchange bias, and point to the need to reexamine the symmetry of the order parameter in monolayer $\mathrm{NbSe}_{2}$. This perspective on the role of magnetism in monolayer $\mathrm{NbSe}_{2}$ will also be crucial to understand and control the superconducting properties of monolayer $\mathrm{NbSe}_{2}$ in the presence of an external magnetic field or with heterostructures between monolayer $\mathrm{NbSe}_{2}$ and magnetic materials.

\section{ACKNOWLEDGMENTS}

The authors thank Maxim Khodas, Roxana Margine, and Rafael Fernandes for useful discussions. D. W. was supported by a National Research Council (NRC) fellowship at the U.S. Naval Research Laboratory. I. I. M. was supported by ONR through Grant No. N00014-20-1-2345.

Note added.-Recently, we became aware of a related article by another group [52], which also indicates that bulk and monolayer $\mathrm{NbSe}_{2}$ are close to a magnetic instability.

\section{APPENDIX A: COMPUTATIONAL METHODS}

Our calculations are based on density-functional theory within the projector-augmented wave method [53] as implemented in the VASP code $[54,55]$ using the generalized gradient approximation defined by the Perdew-BurkeErnzerhof functional [56]. We found that it is essential that $\mathrm{Nb} 5 s^{1}, 4 s^{2}, 4 p^{6}, 4 d^{4}$ electrons and $\mathrm{Se} 4 s^{2}, 4 p^{4}$ electrons are treated as valence. All calculations use a planewave energy cutoff of $400 \mathrm{eV}$. We use a $(18 \times 18 \times 1)$ $\Gamma$-centered $k$-point grid for the monolayer structure and a $(18 \times 18 \times 9) k$-point grid for the bulk structure when performing structural optimization and calculating the electronic structure. The cell shape, volume, and atomic positions of the bulk structure were optimized with the Grimme-D3 van der Waals correction [57] using a force convergence criteria of $5 \mathrm{meV} / \AA$.

To determine the spin susceptibility $\chi$ we used collinear and noncollinear fixed-spin moment calculations (sometimes referred to as the constrained local moments approach). In our collinear FSM calculations we constrain the magnitude of the magnetic moment on the $\mathrm{Nb}$ atom. To determine $\chi$ along a given crystal axes we use noncollinear FSM calculations. In these calculations we constrain the direction (either parallel or perpendicular to the $c$ axis) and the magnitude of the magnetic moment (varying from 0 to $\left.0.8 \mu_{B}\right)$. With these constraints applied we then apply a Lagrange multiplier to the minimization of the total energy. Performing these minimizations as a function of increasing 
magnetic moment and along a given direction allows us to determine the change in energy with respect to the nonmagnetic ground state as a function of the total magnetization $m$ for the bulk and monolayer structures. We then fit our results to an expansion of the total energy as a function of $m$ [Eq. (2)] to determine $\chi$.

The spin susceptibility $\chi$ obtained from FSM calculations is sensitive to the choice in $k$-point grid density, the energy convergence threshold, occupation method, and the number of magnetization values used in the fit to the expansion in the total energy as a function of magnetic moment. To yield converged values of $\chi$ we found it is essential to use a $(28 \times 28 \times 1) \Gamma$-centered $k$-point grid for the monolayer structure and a $(28 \times 28 \times 14) k$-point grid for the bulk structure, an energy convergence threshold of $10^{-8} \mathrm{eV}$, and up to 50 energy versus magnetization points between 0 and $0.8 \mu_{B}$ for all of the FSM calculations. We also found that improved convergence was achieved using the tetrahedron method to determine total energies for both the bulk and monolayer structure (despite the 2D nature of the electronic structure).

For the calculation of the exchange constants within the DLM approximation [58], we used the Korringa-KohnRostokker method within the atomic sphere approximation [59] and the Green's function-based magnetic-force theorem [60]. The implementation of this technique has been described elsewhere [61]. This technique can be considered to be a magnetic analog of the disordered alloys theory based on the coherent potential approximation. Calculations were performed for thirteen nearest-neighbor coordination spheres for five values of the fixed $\mathrm{Nb}$ moments, $0.05 \mu_{B}, 0.1 \mu_{B}, 0.15 \mu_{B}, 0.25 \mu_{B}$, and $0.35 \mu_{B}$, and extrapolated to $M=0$. The nearest neighbor ferromagnetic exchange constant is up to ten times larger than any other exchange constants, while the remaining RKKY interaction is extremely long range and is responsible for weak satellites in Fig. 4. Charge self-consistency was achieved using 147 irreducible $k$ points in the Brillouin zone, and then an extended set of $k$ points (2565) to compute the exchange constants.

\section{APPENDIX B: SINGLET AND TRIPLET PAIRING INTERACTIONS}

To provide an additional derivation of the interactions in Sec. VI, it is useful to consider the contribution of density (that is, electron-phonon) and spin interactions to pairing on the $K$ and $K^{\prime}$ Fermi surfaces. Here, we write these interactions as

$$
\frac{1}{2} \sum_{q} \rho(q) n_{q} n_{-q}+\frac{1}{2} \sum_{i, q} J_{i}(q) S_{i, q} S_{i,-q},
$$

where $n_{q}=\sum_{k, s} c_{k+q / 2, s}^{\dagger} c_{k-q / 2, s}$ and $S_{i, q}=\sum_{q, s, s^{\prime}} c_{k+q / 2, s}^{\dagger} \times$ $\sigma_{i, s, s^{\prime}} c_{k-q / 2, s}$, and $\sigma_{i}$ is a Pauli matrix. For clarity, we have allowed the spin interaction $J_{i}(q)$ to depend upon spin direction $i$, and will later impose isotropy $J_{i}(q)=J(q)$. Equation (B1) assumes interactions take the same form as when inversion symmetry is present, implying we only consider inversion symmetry breaking through single particle interactions. Noting that for sufficiently large Ising spin-orbit coupling, pairing will only occur between states of opposite spin, the contribution of the above interaction toward superconductivity can be written as

$$
\begin{aligned}
& \sum_{k, k^{\prime}}\left[\rho\left(k-k^{\prime}\right)-J_{x}\left(k+k^{\prime}\right)-J_{y}\left(k+k^{\prime}\right)-J_{z}\left(k-k^{\prime}\right)\right] \\
& \quad \times c_{k, \uparrow}^{\dagger} c_{-k, \downarrow}^{\dagger} c_{-k^{\prime}, \downarrow} c_{k^{\prime}, \uparrow},
\end{aligned}
$$

where we have used $\rho(k)=\rho(-k)$ and $J_{i}(k)=J_{i}(-k)$. We now examine Cooper pairs formed from Fermions near the $K$ and $K^{\prime}$ points. To this end, we define operators

$$
\begin{aligned}
& d_{o}^{\dagger}(k)=c_{K+\delta k_{o}, \uparrow}^{\dagger} c_{K^{\prime}-\delta k_{o}, \downarrow}^{\dagger}, \\
& d_{i}^{\dagger}(k)=-c_{K+\delta k_{i}, \downarrow}^{\dagger} c_{K^{\prime}-\delta k_{i}, \uparrow}^{\dagger},
\end{aligned}
$$

where $\delta k_{o}\left(\delta k_{i}\right)$ denote a wave vector on the outer (inner) Fermi pocket at the $K$ point. Here we have introduced the same sign convention for $d_{i}^{\dagger}$ as in Eq. (8) in the main text. For these operators, we find intraband, $g_{i i}$ and $g_{o o}$, and interband, $g_{o i}$ and $g_{i o}$, interactions due to electron-phonon interactions and spin can be defined as

$\sum_{k, k^{\prime}}\left[g_{i i}\left(k, k^{\prime}\right) d_{i}^{\dagger}(k) d_{i}\left(k^{\prime}\right)+g_{o o}\left(k, k^{\prime}\right) d_{o}^{\dagger}(k) d_{o}\left(k^{\prime}\right)\right]$,

$\sum_{\delta k, \delta k^{\prime}}\left[g_{i o}\left(k, k^{\prime}\right) d_{i}^{\dagger}(k) d_{o}\left(k^{\prime}\right)+g_{o i}\left(k, k^{\prime}\right) d_{o}^{\dagger}(k) d_{i}\left(k^{\prime}\right)\right]$,

where

$$
\begin{aligned}
g_{i i}\left(k, k^{\prime}\right)= & \rho\left(\delta k_{i}-\delta k_{i}^{\prime}\right)-J\left(\delta k_{i}-\delta k_{i}^{\prime}\right) \\
& -2 J\left(Q+\delta k_{i}+\delta k_{i}^{\prime}\right), \\
g_{o o}\left(k, k^{\prime}\right)= & \rho\left(\delta k_{o}-\delta k_{o}^{\prime}\right)-J\left(\delta k_{o}-\delta k_{o}^{\prime}\right) \\
& -2 J\left(Q+\delta k_{o}+\delta k_{o}^{\prime}\right),
\end{aligned}
$$

$$
\begin{aligned}
g_{i o}\left(k, k^{\prime}\right)= & g_{o i}\left(k, k^{\prime}\right)=\rho\left(Q+\delta k_{i}+\delta k_{o}^{\prime}\right) \\
& -J\left(Q+\delta k_{i}+\delta k_{o}^{\prime}\right)-2 J\left(\delta k_{i}-\delta k_{o}^{\prime}\right),
\end{aligned}
$$

where $Q=2 K$ and we have imposed spin isotropy $J_{i}(k)=$ $J(k)$. From this expression, and taking $J(Q+\delta k) \approx 0$, the coupling constants found in Table II can be readily deduced.

It is instructive to consider the limit $\delta k_{i}=\delta k_{o} \rightarrow 0$, then, when $J(Q) \approx 0, g_{i i}=g_{o o}=-g^{p}(0)+g^{s}(0)$ and $g_{i o}=$ $g_{o i}=-g^{p}(Q)+2 g^{s}(0)$, where the constants $g^{p}(0)$ and 
$g^{p}(Q)$ are defined to be positive, corresponding to attractive electron-phonon interactions, and $g^{s}(0)$ is negative corresponding to repulsive ferromagnetic interactions. In this case, a pure singlet state corresponds to the operator $\left[d_{i}(k)+d_{o}(k)\right] / \sqrt{2}$ for which the interaction is

$$
v_{s}=-g^{p}(0)-g^{p}(Q)-3 g^{s}(0) .
$$

A pure triplet case corresponds to the operator $\left[d_{i}(k)-d_{o}(k)\right] / \sqrt{2}$, for which the interaction is

$$
v_{t}=-g^{p}(0)+g^{p}(Q)+g^{s}(0) .
$$

These expressions reveal how spin fluctuations strongly suppress the spin-singlet state and enhance the spin-triplet state. Notice that once the spin fluctuations become sufficiently strong, that is $\left|g^{p}(Q)\right|<2\left|g^{s}(0)\right|$, the triplet solution will have a higher $T_{c}$ than the singlet solution.

\section{APPENDIX C: CRITICAL FIELD ANISOTROPY FOR A NODAL FERMI SURFACE}

As we discuss in Sec. V, the third Fermi pocket, around $\Gamma$, has zero spin-orbit coupling (SOC) splitting along the $\Gamma-M$ and $\Gamma-M^{\prime}$ directions. Here, we rederive the expression for the spin susceptibility for this band topology that accounts for the nodes along these directions.

Assuming that the SO splitting varies angularly as $\lambda \cos (3 \varphi)$, we derive the susceptibility, $d m / d H_{x}$, for an in-plane magnetic field applied along $\hat{x}$,

$$
m=\frac{H}{2 \pi \lambda} \int_{0}^{2 \pi} \frac{d \varphi}{\sqrt{\cos ^{2} \varphi+\zeta^{2}}},
$$

where $\lambda$ is the maximal SOC splitting on this Fermi contour and $\zeta=H / \lambda$. This gives the same Pauli expression as before, but with a logarithmic correction:

$$
\frac{d m}{d H_{x}}=\chi_{\text {Pauli }}\left(1+\frac{3}{4} \zeta^{2} \log \zeta\right)
$$

In the superconducting state, $\lambda \cos (3 \varphi)$ is replaced by $\sqrt{\lambda^{2} \cos ^{2}(3 \varphi)+\Delta^{2}}$, where $\Delta$ is the average superconducting gap along the $\Gamma$ contour and $\zeta$ defined above is replaced with $\zeta=\sqrt{\Delta^{2}+H^{2}} / \lambda$. Then in the superconducting state,

$$
\begin{aligned}
m & =\frac{1}{2 \pi} \int_{0}^{2 \pi} \frac{H d \varphi}{\sqrt{\lambda^{2} \cos ^{2} \varphi+\Delta^{2}+H^{2}}} \\
& =\frac{m}{2 \pi \lambda} \int_{0}^{2 \pi} \frac{d \varphi}{\sqrt{\cos ^{2} \varphi+\zeta^{2}}} .
\end{aligned}
$$

Upon integration, we get a logarithmic correction to the susceptibility in the superconducting state, namely,

$$
\frac{d m}{d H}=\chi_{\text {Pauli }}\left(1+\frac{1}{4} \zeta_{0}^{2} \log \zeta_{0}\right)
$$

where $\zeta_{0}=\Delta / \lambda$. That is to say, the anisotropy of the thermodynamic critical field is not infinite, but, roughly,

$$
\left|\frac{N_{\Gamma}+N_{K}}{N_{\Gamma} \zeta_{0}^{2} \log \zeta_{0}}\right|,
$$

where $N_{\Gamma(K)}$ is the DOS around the $\Gamma$ contour and $N_{K}$ is the total DOS around the $K$ and $K^{\prime}$ pockets. While this factor is formally finite, it is a very large number of the order of $10^{3}$. Instead, other factors, such as a substrate induced Rashba spin-orbit coupling [27] and impurity scattering [26], are more important in limiting the anisotropy of the critical field.

[1] M. Leroux, I. Errea, M. Le Tacon, S.-M. Souliou, G. Garbarino, L. Cario, A. Bosak, F. Mauri, M. Calandra, and P. Rodière, Strong Anharmonicity Induces Quantum Melting of Charge Density Wave in $2 \mathrm{H}-\mathrm{NbSe}_{2}$ Under Pressure, Phys. Rev. B 92, 140303(R) (2015).

[2] B. Guster, C. Rubio-Verdu, R. Robles, J. Zaldivar, P. Dreher, M. Pruneda, J. A. Silva-Guillen, D.-J. Choi, J. I. Pascual, M. M. Ugeda, P. Orderjon, and E. Canadell, Coexistence of Elastic Modulations in the Charge Density Wave State of $2 \mathrm{H}$-NbSe${ }_{2}$, Nano Lett. 19, 3027 (2019).

[3] X. Xi, L. Zhao, Z. Wang, H. Berger, L. Forró, J. Shan, and K. F. Mak, Strongly Enhanced Charge-Density-Wave Order in Monolayer $\mathrm{NbSe}_{2}$, Nat. Nanotechnol. 10, 765 (2015).

[4] M. M. Ugeda, A. J. Bradley, Y. Zhang, S. Onishi, Y. Chen, W. Ruan, C. Ojeda-Aristizabal, H. Ryu, M. T. Edmonds, H.-Z. Tsai, A. Riss, S.-K. Mo, D. Lee, A. Zettl, Z. Hussain, Z.-X. Shen, and M. Crommie, Characterization of Collective Ground States in Single-Layer $\mathrm{NbSe}_{2}$, Nat. Phys. 12, 92 (2016).

[5] R. Bianco, L. Monacelli, M. Calandra, F. Mauri, and I. Errea, Weak Dimensionality Dependence of the Charge Density Wave Transition in $\mathrm{NbSe}_{2}$, arXiv:2004.08147.

[6] R. Frindt, Superconductivity in Ultrathin $\mathrm{NbSe}_{2}$ Layers, Phys. Rev. Lett. 28, 299 (1972).

[7] M. Bonilla, S. Kolekar, Y. Ma, H. C. Diaz, V. Kalappattil, R. Das, T. Eggers, H. R. Gutierrez, M.-H. Phan, and M. Batzill, Strong Room-Temperature Ferromagnetism in $\mathrm{VSe}_{2}$ Monolayers on van der Waals Substrates, Nat. Nanotechnol. 13, 289 (2018).

[8] D. E. Moncton, J. Axe, and F. DiSalvo, Neutron Scattering Study of the Charge-Density Wave Transitions in $2 \mathrm{H}-\mathrm{TaSe}_{2}$ and $2 \mathrm{H}-\mathrm{NbSe}_{2}$, Phys. Rev. B 16, 801 (1977).

[9] S. Foner and E. McNiff, Jr., Upper Critical Fields of Layered Superconducting $\mathrm{NbSe}_{2}$ at Low Temperature, Phys. Lett. A 45, 429 (1973).

[10] E. Khestanova, J. Birkbeck, M. Zhu, Y. Cao, G. Yu, D. Ghazaryan, J. Yin, H. Berger, L. Forro, T. Taniguchi, K. Watanabe, R. Gorbachev, A. Mishchenko, A. Geim, and I. Grigorieva, Unusual Suppression of the Superconducting 
Energy Gap and Critical Temperature in Atomically Thin $\mathrm{NbSe}_{2}$, Nano Lett. 18, 2623 (2018).

[11] Y. Noat, J. A. Silva-Guillén, T. Cren, V. Cherkez, C. Brun, S. Pons, F. Debontridder, D. Roditchev, W. Sacks, L. Cario et al., Quasiparticle Spectra of $2 \mathrm{H}-\mathrm{NbSe}_{2}$ : Two-Band Superconductivity and the Role of Tunneling Selectivity, Phys. Rev. B 92, 134510 (2015).

[12] M. Calandra, I. Mazin, and F. Mauri, Effect of Dimensionality on the Charge-Density Wave in Few-Layer $2 \mathrm{H}-\mathrm{NbSe}_{2}$, Phys. Rev. B 80, 241108(R) (2009).

[13] C.-S. Lian, C. Si, and W. Duan, Unveiling Charge-Density Wave, Superconductivity, and Their Competitive Nature in Two-Dimensional $\mathrm{NbSe}_{2}$, Nano Lett. 18, 2924 (2018).

[14] K. Cho, M. Kończykowski, S. Teknowijoyo, M. A. Tanatar, J. Guss, P. Gartin, J. M. Wilde, A. Kreyssig, R. McQueeney, A. I. Goldman et al., Using Controlled Disorder to Probe the Interplay between Charge Order and Superconductivity in $\mathrm{NbSe}_{2}$, Nat. Commun. 9, 2796 (2018).

[15] X. Xi, Z. Wang, W. Zhao, J.-H. Park, K. T. Law, H. Berger, L. Forró, J. Shan, and K. F. Mak, Ising Pairing in Superconducting $\mathrm{NbSe}_{2}$ Atomic Layers, Nat. Phys. 12, 139 (2016).

[16] C. Sergio, M. R. Sinko, D. P. Gopalan, N. Sivadas, K. L. Seyler, K. Watanabe, T. Taniguchi, A. W. Tsen, X. Xu, D. Xiao, and B. Hunt, Tuning Ising Superconductivity with Layer and Spin-Orbit Coupling in Two-Dimensional Transition-Metal Dichalcogenides, Nat. Commun. 9, 1427 (2018).

[17] C.-w. Cho, J. Lyu, T. Han, C. Y. Ng, Y. Gao, G. Li, M. Huang, N. Wang, and R. Lortz, Distinct Nodal and Nematic Superconducting Phases in the 2D Ising Superconductor $\mathrm{NbSe}_{2}$, arXiv:2003.12467.

[18] D. Shaffer, J. Kang, F. J. Burnell, and R. M. Fernandes, Crystalline Nodal Topological Superconductivity and Bogolyubov Fermi Surfaces in Monolayer $\mathrm{NbSe}_{2}$, Phys. Rev. B 101, 224503 (2020).

[19] B. T. Zhou, N. F. Q. Yuan, H.-L. Jiang, and K. T. Law, Ising Superconductivity and Majorana Fermions in Transition-Metal Dichalcogenides, Phys. Rev. B 93, 180501(R) (2016).

[20] S. Głodzik and T. Ojanen, Engineering Nodal Topological Phases in Ising Superconductors by Magnetic Superstructures, New J. Phys. 22, 013022 (2020).

[21] A. Hamill, B. Heischmidt, E. Sohn, D. Shaffer, K.-T. Tsai, X. Zhang, X. Xi, A. Suslov, H. Berger, L. Forró, F. Burnell, J. Shan, K. F. Mak, R. Fernandes, K. Wang, and V. Pribiag, Unexpected Two-Fold Symmetric Superconductivity in Few-Layer $\mathrm{NbSe}_{2}$, arXiv:2004.02999.

[22] S. Kezilebieke, M. N. Huda, V. Vaňo, M. Aapro, S. C. Ganguli, O. J. Silveira, S. Głodzik, A. S. Foster, T. Ojanen, and P. Liljeroth, Topological Superconductivity in a Designer Ferromagnet-Superconductor van der Waals Heterostructure, arXiv:2002.02141.

[23] J. Lu, O. Zheliuk, I. Leermakers, N. F. Yuan, U. Zeitler, K. T. Law, and J. Ye, Evidence for Two-Dimensional Ising Superconductivity in Gated $\mathrm{MoS}_{2}$, Science 350, 1353 (2015).

[24] J. Cui, P. Li, J. Zhou, W.-Y. He, X. Huang, J. Yi, J. Fan, Z. Ji, X. Jing, F. Qu, Z. G. Cheng, C. Yang, L. Lu, K. Suenaga, J. Liu, K. T. Law, J. Lin, Z. Liu, and G. Liu, Transport Evidence of Asymmetric Spin-Orbit Coupling in Few-Layer Superconducting $1 T_{d}-\mathrm{MoTe}_{2}$, Nat. Commun. 10, 1 (2019).
[25] D. Xiao, G.-B. Liu, W. Feng, X. Xu, and W. Yao, Coupled Spin and Valley Physics in Monolayers of $\mathrm{MoS}_{2}$ and Other Group-VI Dichalcogenides, Phys. Rev. Lett. 108, 196802 (2012).

[26] D. Möckli and M. Khodas, Ising Superconductors: Interplay of Magnetic Field, Triplet Channels, and Disorder, Phys. Rev. B 101, 014510 (2020).

[27] W.-Y. He, B. T. Zhou, J. J. He, N. F. Yuan, T. Zhang, and K. Law, Magnetic Field Driven Nodal Topological Superconductivity in Monolayer Transition Metal Dichalcogenides, Commun. Phys. 1, 40 (2018).

[28] S. J. Youn, M. H. Fischer, S. H. Rhim, M. Sigrist, and D. F. Agterberg, Role of Strong Spin-Orbit Coupling in the Superconductivity of the Hexagonal Pnictide SrPtAs, Phys. Rev. B 85, 220505(R) (2012).

[29] P. A. Frigeri, D. F. Agterberg, A. Koga, and M. Sigrist, Superconductivity without Inversion Symmetry: MnSi versus $\mathrm{CePt}_{3} \mathrm{Si}$, Phys. Rev. Lett. 92, 097001 (2004).

[30] M. Smidman, M. B. Salamon, H. Q. Yuan, and D. F. Agterberg, Superconductivity and Spin-Orbit Coupling in Non-Centrosymmetric Materials: A Review, Rep. Prog. Phys. 80, 036501 (2017).

[31] M. Calandra, P. Barone, and F. Mauri (private communication).

[32] C. Heil, S. Poncé, H. Lambert, M. Schlipf, E. R. Margine, and F. Giustino, Origin of Superconductivity and Latent Charge Density Wave in $\mathrm{NbS}_{2}$, Phys. Rev. Lett. 119, 087003 (2017).

[33] M. Iavarone, R. Di Capua, G. Karapetrov, A. E. Koshelev, D. Rosenmann, H. Claus, C. D. Malliakas, M. G. Kanatzidis, T. Nishizaki, and N. Kobayashi, Effect of Magnetic Impurities on the Vortex Lattice Properties in $\mathrm{NbSe}_{2}$ Single Crystals, Phys. Rev. B 78, 174518 (2008).

[34] L. Boeri, M. Calandra, I. I. Mazin, O. V. Dolgov, and F. Mauri, Effects of Magnetism and Doping on the ElectronPhonon Coupling in $\mathrm{BaFe}_{2} \mathrm{As}_{2}$, Phys. Rev. B 82, 020506(R) (2010).

[35] D. Fay and J. Appel, Coexistence of p-State Superconductivity and Itinerant Ferromagnetism, Phys. Rev. B 22, 3173 (1980).

[36] Y. Xu, X. Liu, and W. Guo, Tensile Strain Induced Switching of Magnetic States in $\mathrm{NbSe}_{2}$ and $\mathrm{NbS}_{2}$ Single Layers, Nanoscale 6, 12929 (2014).

[37] A. B. Vorontsov, I. Vekhter, and M. Eschrig, Surface Bound States and Spin Currents in Noncentrosymmetric Superconductors, Phys. Rev. Lett. 101, 127003 (2008).

[38] C.-K. Lu and S. Yip, Zero-Energy Vortex Bound States in Noncentrosymmetric Superconductors, Phys. Rev. B 78, 132502 (2008).

[39] P. A. Frigeri, D. F. Agterberg, and M. Sigrist, Spin Susceptibility in Superconductors without Inversion Symmetry, New J. Phys. 6, 115 (2004).

[40] M. Marezio, P. Dernier, A. Menth, and G. Hull, Jr., The Crystal Structure of $\mathrm{NbSe}_{2}$ at $15^{\circ} \mathrm{K}$, J Solid State Chem. 4, 425 (1972).

[41] L. Mattheiss, Energy Bands for $2 \mathrm{H}-\mathrm{NbSe}_{2}$ and $2 \mathrm{H}-\mathrm{MoS}_{2}$, Phys. Rev. Lett. 30, 784 (1973).

[42] M. D. Johannes, I. I. Mazin, and C. A. Howells, FermiSurface Nesting and the Origin of the Charge-Density Wave in $\mathrm{NbSe}_{2}$, Phys. Rev. B 73, 205102 (2006). 
[43] J. Á. Silva-Guillén, P. Ordejón, F. Guinea, and E. Canadell, Electronic Structure of $2 \mathrm{H}-\mathrm{NbSe}_{2}$ Single-Layers in the CDW State, 2D Mater. 3, 035028 (2016).

[44] T. Moriya, Spin Fluctuations in Itinerant Electron Magnetism (Springer Science \& Business Media, 2012), Vol. 56, https://www.springer.com/gp/book/9783642825019.

[45] P. Larson, I. I. Mazin, and D. J. Singh, Magnetism, Critical Fluctuations, and Susceptibility Renormalization in Pd, Phys. Rev. B 69, 064429 (2004).

[46] B. Kim, S. Khmelevskyi, I. I. Mazin, D. F. Agterberg, and C. Franchini, Anisotropy of Magnetic Interactions and Symmetry of the Order Parameter in Unconventional Superconductor $\mathrm{Sr}_{2} \mathrm{RuO}_{4}$, npj Quantum Mater. 2, 1 (2017).

[47] G. L. Krasko, Metamagnetic Behavior of fcc Iron, Phys. Rev. B 36, 8565 (1987).

[48] O. Andersen, J. Madsen, U. Poulsen, O. Jepsen, and J. Kollar, Magnetic Ground State Properties of Transition Metals, Physica (Amsterdam) 86-88B+C, 249 (1977).

[49] D. Parker, M. G. Vavilov, A. V. Chubukov, and I. I. Mazin, Coexistence of Superconductivity and a Spin-Density Wave in Pnictide Superconductors: Gap Symmetry and Nodal Lines, Phys. Rev. B 80, 100508(R) (2009).

[50] I. I. Mazin and V.P. Antropov, Electronic Structure, Electron-Phonon Coupling, and Multiband Effects in $\mathrm{MgB}_{2}$, Physica (Amsterdam) 385C, 49 (2003).

[51] E. R. Margine et al. (to be published).

[52] S. Divilov, W. Wan, P. Dreher, M. M. Ugeda, and F. Ynduráin, Interplay between Magnetism and Charge Instabilities in Layered $\mathrm{NbSe}_{2}$, arXiv:2005.06210.
[53] P. E. Blöchl, Projector Augmented-Wave Method, Phys. Rev. B 50, 17953 (1994).

[54] G. Kresse and J. Hafner, Ab Initio Molecular Dynamics for Liquid Metals, Phys. Rev. B 47, 558 (1993).

[55] G. Kresse and J. Furthmüller, Efficient Iterative Schemes for Ab Initio Total-Energy Calculations Using a Plane-Wave Basis Set, Phys. Rev. B 54, 11169 (1996).

[56] J. P. Perdew, K. Burke, and M. Ernzerhof, Generalized Gradient Approximation Made Simple, Phys. Rev. Lett. 77, 3865 (1996).

[57] S. Grimme, J. Antony, S. Ehrlich, and H. Krieg, A Consistent and Accurate Ab Initio Parametrization of Density Functional Dispersion Correction (DFT-D) for the 94 Elements H-Pu, J. Chem. Phys. 132, 154104 (2010).

[58] B. Gyorffy, A. Pindor, J. Staunton, G. Stocks, and H. Winter, A First-Principles Theory of Ferromagnetic Phase Transitions in Metals, J. Phys. F 15, 1337 (1985).

[59] A. Ruban and H. L. Skriver, Calculated Surface Segregation in Transition Metal Alloys, Comput. Mater. Sci. 15, 119 (1999).

[60] A. I. Liechtenstein, M. Katsnelson, V. Antropov, and V. Gubanov, Local Spin Density Functional Approach to the Theory of Exchange Interactions in Ferromagnetic Metals and Alloys, J. Magn. Magn. Mater. 67, 65 (1987).

[61] A. V. Ruban, S. Shallcross, S. I. Simak, and H. L. Skriver, Atomic and Magnetic Configurational Energetics by the Generalized Perturbation Method, Phys. Rev. B 70, 125115 (2004). 